\title{
La ciencia como empresa social: su evaluación desde la bibliometría
}

\author{
Guillermo Andrés Pérez Reyes \\ Universidad Politécnica Territorial de Lara Andrés Eloy Blanco - UPTAEB, Venezuela
}

Ailín Martínez Rodríguez

Universidad de La Habana - UH, Cuba

REVIEW

\begin{abstract}
Resumen
El propósito de este artículo es realizar una revisión teórica de la Ciencia enmarcando a la misma como empresa social, asimismo se relaciona la bibliometría como herramienta fundamental para la evaluación de ésta. Este artículo se apoya en una investigación documental. Se concluye que La ciencia como empresa social debe dar respuestas a las necesidades de la sociedad a partir de sus contribuciones y que la misma puede ser medida a partir del uso de indicadores bibliométricos.
\end{abstract}

Palabras clave

Ciencia ; Empresa social ; Evaluación de la Ciencia ; Bibliometría ; Indicadores bibliométricos

\section{Science as social enterprise: its evaluation from bibliometrics}

\section{Abstract}

The purpose of this article is to review theoretical framing of science to it as a social enterprise also bibliometrics as a fundamental tool for evaluating it relates. This article is based on documentary research. We conclude that science as a social enterprise to provide answers to the needs of society from their contributions and that it can be measured through the use of bibliometric indicators.

Keywords

Science, Social enterprise ; Evaluation of Science ; Bibliometrics ; Bibliometric indicators

\section{Introducción}

La ciencia durante muchos años ha sido un elemento importantísimo de la actividad humana, ya que la misma, es un sistema de conocimientos en constante desarrollo, cuya veracidad se comprueba a través de la práctica social. No obstante, delimitar o definir qué se entiende por ciencia no logra ser algo sencillo y simple, cuyas primeras aproximaciones pueden darse conociendo la raíz de la palabra, pero más allá de plantearse las primeras nociones, la ciencia en pleno siglo XXI contribuye significativamente con la sociedad, por lo que la denominación de empresa social es determinante para conocer el papel que juegan la ciencia, los científicos y la sociedad. 
La ciencia como actividad social posee fuertes implicaciones que impactan significativamente en el progreso científico y tecnológico de un país, sin embargo, el desarrollo de políticas científicas es uno de los principales intereses de los gobernantes para así distribuir eficazmente los recursos económicos, asimismo la actividad científica a partir de las producciones intelectuales que genera, se convierten en un insumo importante para constatar el crecimiento de las áreas de conocimientos e identificar los frentes de investigación.

La aplicación de los métodos cuantitativos al análisis de la producción científica, desde el punto de vista de las Ciencias de la Información se encuentra enmarcada dentro de los Estudios Métricos de la Información, término genérico que se emplea para agrupar las principales metrías de información tales como: la Bibliometría, la Cienciometría, la Informetría, entre otros, todas utilizadas para definir las características de los procesos de la comunicación escrita y la naturaleza y desarrollo de las disciplinas científicas a partir de la aplicación de métodos estadísticos y matemáticos.

\section{La ciencia como empresa social}

El término "ciencia" deriva etimológicamente del latín scire, que significa "saber", "conocer". Sin embargo, ya desde la antigüedad, este vocablo estuvo reservado para aludir a una forma más elevada de conocimiento, distinta del conocimiento común o vulgar (Diccionario de Ciencias Sociales, 1975, p. 377).

En sentido general la palabra ciencia hace referencia a todo tipo de saber, a estar informado, por lo que literalmente equivale a conocimiento.

La ciencia tiene una mirada ambivalente producto de las diferentes posturas con la cual se ha pretendido definirla. En palabras de Latour (1983), la ciencia posee dos caras (las dos caras de Jano): ciencia establecida y ciencia en acción, la primera de ella se enaltece por ser un enorme símbolo de poder y una exhibición de los logros, y al mismo tiempo un modelo de referencia; y la ciencia en acción está en la frontera del conocimiento, luchando por establecer nuevas verdades en un territorio resbaladizo (Maltrás, 2001, p. 22).

Por otro lado es importante señalar que autores muy renombrados en el estudio de la ciencia han marcado su postura a la hora de trazar una línea de demarcación de manera de encontrar un criterio de distinguir qué es o no es ciencia. Entre estos autores y sus respuestas al "problema de la demarcación de la ciencia" están: Popper y Lakatos (modelo racionalista de la actividad científica), Feyerabend (el anrquísta/dadaísta), Bunge (el materialista/racionalista) Cappra (la fusión de la ciencia occidental con la sabiduría oriental), entre otros (Ander-Egg, 2003, p. 50).

Por lo tanto, la ciencia en el transcurrir del tiempo ha ido de la mano con los nuevos aportes que numerosos científicos han brindado para definir la ciencia bajo las realidades y verdades que descubren en sus épocas, sin embargo, en el recorrido que se ha realizado por develar la concepción de qué se entiende por ciencia puede reducirse, como señalan sus autores, a las siguientes nociones tomando en cuenta las principales características que la distinguen:

Ander-Egg (2003) cuando enuncia su noción de ciencia hace referencia a:

Un conjunto de conocimientos racionales, de tipo conjetural que pueden ser verdaderos o falsos (nunca se tiene certeza absoluta), y que se obtienen de una manera metódica y se verifican en su validez y fiabilidad mediante la contrastación empírica. Este cuerpo de conocimientos, orgánicamente sistematizados dentro de cada ciencia, hace referencia a hechos y fenómenos de una misma naturaleza. A medida que la ciencia evoluciona, ya sea por la adquisición de nuevos conocimientos, la utilización de mejores métodos y técnicas de investigación, el cambio de paradigma o nuevas reflexiones de los científicos, estos conocimientos se modifican y corrigen, lo que implica la posibilidad de biodegradabilidad de todo enunciado científico. Toda ciencia, además utiliza un lenguaje o vocabulario que le es propio y que es susceptible de ser transmitido (p.58).

Asimismo en el área de las Ciencias de la Información, Rendón (2005) señala que:

la ciencia nos da testimonio de que existe una forma específica de conocer el mundo, y que se caracteriza por ser verdadera, justificada, que utiliza herramientas del pensamiento racional (conceptos, juicios y raciocinios), que tiende a la generalidad y busca regularidades, que está 


\begin{abstract}
plasmada en teorías como sistemas de enunciados con una estructura determinada, y que tiene la
\end{abstract} función de describir, explicar, predecir y comprender la realidad (p.38).

Como se ha afirmado en los párrafos anteriores, la noción de ciencia es vista de una manera muy lineal, quizás muy propio de la ciencia hegemónica, que durante muchos años ha marcado el cómo hacer las cosas y el cómo explicarlas a partir de sus tradicionales métodos, a pesar que hoy, la variedad metodológica y los métodos que se aplican responden a una realidad más enmarcada al sujeto que al objeto, sobre todo en las Ciencias Sociales, ahora bien, el punto que interesa resaltar no está en la metodología y los métodos que aplique la ciencia sino que en las definiciones en torno a la ciencia no se aprecia en forma explícita la función social que debe cumplir para dar respuesta a las necesidades de la sociedad y por ende poder denominar a la ciencia como empresa social.

Uno de los autores más significativos en los temas de la función social que debe cumplir la ciencia es, Jhon D. Bernal, quien se preocupó siempre del pasado, el presente y el futuro de la historia de la ciencia, y de sus implicaciones sociales. Dentro de las publicaciones más importante se encuentra su libro titulado La Función Social de la Ciencia cuyo escrito cambió la concepción de la ciencia, el científico y su relación con la sociedad.

Bernal es uno de los principales pioneros en establecer la función social que la ciencia debe cumplir, por un lado enfatiza la responsabilidad moral que el científico debe asumir, y de "no jugar" el papel "importante" que desarrolló en la Primera y Segunda Guerra Mundial donde el armamento y la fabricación de la bomba nuclear e incluyendo la de hidrógeno fueron ampliamente responsables de los males y catástrofes de la época. Por otro lado señala que es por medio de la ciencia que toda nuestra civilización está siendo transformada y que la misma se está desarrollando a grandes saltos y de un modo visible por todos (Bernal, 1979).

Bernal cierra con la siguiente idea en cuanto al papel que debe jugar la triada científico, ciencia y responsabilidad social:

La alternativa no es la irresponsabilidad, sino una responsabilidad social más consciente y activa mediante la cual la ciencia pueda, por una parte hacer una contribución explícita a la planificación de la industria, la agricultura y la medicina para fines que el científico pueda aprobar plenamente, y por otra, extenderse y trasformarse hasta convertirse en parte integral de la vida y del trabajo de todos (p.26).

Sin embargo, Bernal (1979), en su intento de develar la noción de ciencia o por lo menos, los aspectos más importantes, señala que "la ciencia está cambiando muy rápidamente en la medida que es uno de los logros más recientes de la humanidad" por lo que, una definición de ciencia "no puede aplicarse estrictamente a una actividad humana que en sí misma es sólo un aspecto inseparable del proceso único e irrepetible de la evolución social” (p. 27).

Entre los aspectos más importantes que este autor señala deben contemplarse en la ciencia están:

- La ciencia como institución.

- Los métodos de la ciencia.

- La ciencia como medio de producción.

- La ciencia natural como fuente de idea.

- Interacción de ciencia y sociedad.

- La tradición acumulativa de la ciencia, de especial significación para este trabajo, teniendo en cuenta que la ciencia es un cuerpo de conocimiento en constante desarrollo formado por fragmentos de las reflexiones, las ideas, experiencias y acciones de una amplia corriente de trabajadores y pensadores. Los científicos para poner en marcha sus investigaciones deben de contar con un inmenso fondo de experiencias y conocimientos anteriores que les permitan poner en marcha las nuevas investigaciones y así contribuir con el fondo acumulativo de conocimientos. Por lo tanto en la ciencia siempre hay nuevos descubrimientos, nuevas leyes y nuevas teorías. 
Hasta ahora se han señalado las diferentes acepciones que la ciencia ha tenido durante diferentes etapas señalando que la noción de ciencia depende solo de la evolución social y la importancia de las acciones de los científicos para cumplir con las necesidades de la sociedad y de sus propias áreas de conocimientos.

Por otro lado, la ciencia está conformada por una comunidad científica que construye colectivamente para beneficio de un conglomerado profesional y una sociedad ávida de crecer de la mano con la ciencia desde el punto de vista tecnológico, social, educativo, etc.

Al hablar de comunidad científica insoslayablemente no se puede dejar de mencionar a Thomas Kunh que con sus pensamientos revolucionaron la nueva forma de denominar la ciencia, "paradigma", sin embargo, la opinión compartida entre científicos, sociólogos e historiadores, según Kunh (1971) en su posdata de 1969, una comunidad científica:

Consiste en quienes practican una especialidad científica. Hasta un grado no igualado en la mayoría de los otros ámbitos, han tenido una educación y una iniciación profesional similares. En el proceso, han absorbido la misma bibliografía técnica y sacado muchas lecciones idénticas de ella (p.272).

Asimismo, Kuhn (1971, citado por Almeida, 2010) plantea que las comunidades científicas se constituyen principalmente:

Por la fundación de publicaciones periódicas, sociedades profesionales, la exigencia y apropiación de un espacio diferenciado del conjunto de la ciencia relacionado directamente con la primera aceptación de un paradigma y la absorción de la misma bibliografía, cuyos límites temáticos constituyen las fronteras disciplinarias (p.17).

A partir de los aspectos que hasta ahora se han discutido en torno a la ciencia, se ha destacado la función social que debe tener la misma en pro del beneficio de la sociedad a partir del contexto y la realidad que viven. En este orden de ideas, en cada una de las posturas teóricas implícitamente se encuentran los elementos más distintivos para denominar a la ciencia como empresa social: la ciencia como institución, la ciencia como medio de producción y la interacción de ciencia y sociedad (Bernal, 1979); la biodegradabilidad de todo enunciado científico a partir de las nuevas reflexiones de los científicos (Ander-Egg, 2003); y la comunidad científica (Kuhn, 1971), donde esta última, juega el papel más importante porque son ellos quienes impulsan a la ciencia a través de sus producciones de investigación.

Ísita (2000) señala que "la ciencia es una empresa social en virtud de que las observaciones y teorías deben ser conocidas, aceptadas o refutadas, en principio por una comunidad social, la comunidad de investigación científica" ( $p$ 211).

La ciencia como empresa social depende del contexto social donde se desarrolla, asimismo, necesita que se le asignen recursos, y la misma no deja de ser una empresa productora de información que requiere de insumos y como generadora de resultados requiere ser evaluada para medir su rendimiento y su impacto en la sociedad.

En este sentido Ciencia como empresa social "dentro de una red más vasta de estructura social y conocimiento público, es el punto de partida para abordar el tema de la evaluación de la actividad científica". Por lo tanto los elementos más importantes para el desarrollo de la ciencia "dependen directamente de la comunidad científica que los produce, los difunde y los "consume", por lo que se hace necesario saber cuáles son los procesos que producen ese conocimiento, procesos susceptibles de ser analizados a partir de métodos cuantitativos complementarios de otros" (Lascurain, 2006, p. 9).

\section{Evaluación de la ciencia como empresa social a partir de la bibliométría}

La evaluación de la ciencia es una actividad que surge a partir del impacto que generó la Primera Guerra Mundial. A pesar de los grandes progresos que la actividad científica había logrado, aún no era capaz de solucionar problemas sociales importantes como la mala distribución de los ingresos, la subalimentación y las diversas enfermedades que afectaban a vastos sectores de la población mundial, pero que si era capaz de producir armas de guerra cada vez 
más poderosas, es por ello, y tal como se ha dejado claro en el acápite anterior, la ciencia debía dar una satisfacción a la sociedad (Velho, citado por Nadia, 2000, p.10).

Por otro lado, a partir de la Segunda Guerra Mundial se incrementa considerablemente la producción científica, lo que permitió el desarrollo de nuevas disciplinas y la participación de nuevos científicos, por lo cual, paralelamente la producción científica se incrementó a través de los diversos eventos, patentes y otros nuevos documentos (Chaviano, 2004).

La ciencia es evaluación (Van Raan, 1990) y la evaluación a su vez es un proceso social, de manera que la forma de llevarla a cabo resulta determinante para la obtención de sus resultados (Georghiou, 1990 citado por Ruiz, 2002, p. 9).

La evaluación según Sanz Menendez (2004) es: "...un instrumento para determinar la asignación de recursos, un mecanismo para cambiar estructuras organizativas y definir nuevos incentivos, o para evaluar los resultados en ciertas áreas científicas en relación con las necesidades nacionales" (p. 2).

Según López Yépez (1999) la evaluación "es la valoración cualitativa y cuantitativa, así como la crítica objetiva de todos los elementos que constituyen el proceso de la investigación científica con ayuda de métodos adecuados" (p. 203).

Según Van Raan (1996) "la evaluación de las actividades de investigación es importante para promover la investigación en términos de valor y calidad”. En este orden de ideas, este autor señala que los términos valor y calidad juegan un papel importante, "el término valor se relaciona con la verdad, desde el punto de vista de la práctica científica (de modo que lo más valioso es conseguir la verdad de la forma más eficiente posible) y con la utilidad, desde el punto de vista de la administración (lo más valioso es aquello que, además de ser verdad, es útil)" y en cuanto a la calidad "este es un concepto complejo que tiene connotaciones sociales, filosóficas, morales y éticas (Pacheco y Milanés, 2009, p. 4).

Sin embargo, la tarea de evaluar es ardua e imposible en exactitud debido a que la tarea científica no es químicamente pura pues está sometida a circunstancias ambientales como el poder de la financiación, la existencia de materiales humanos en mayor y menor medida, las relaciones entre política y ciencia e incluso las relaciones entre los propios científicos y entre las propias instituciones de investigación (López Yépez, 2000, p. 201).

Es por ello, que en las producciones científicas se encuentran el principal insumo para la evaluación de la ciencia, ya que la misma "es considerada como la parte materializada del conocimiento generado" (Piedra y Martínez, 2007, p. 33). Esta materialización no es más que los "resultados de investigación científica de autores, instituciones, regiones, países en general o bien en áreas temáticas específicas" plasmados en "libros, artículos de periódicos y otras modalidades de publicaciones impresas, digitales o electrónicas" (Oliveira y Moraes, citado por Becerril y otros, 2012, p.56).

En esta línea de pensamiento, evaluar los resultados de investigación, se convierte en la tarea principal de los principales sectores científicos, instituciones y gobiernos, ya que, estas instituciones o sectores requieren "mantener un control en las asignaciones de los recursos, tanto humanos como materiales" por lo cual ha incentivado enormemente que "la evaluación de la investigación adquiera un papel relevante y se constituya como una actividad fundamental de la política científica actual" y poder contribuir a garantizar a la sociedad "el correcto empleo de los recursos" (Pacheco y Milanés 2009, p. 3).

Por otro lado, autores como Bellavista y otros (Lascurain, 2006, p.10) señalan que la evaluación de la actividad científica contempla tres procesos: evaluación "ex ante" (appraisal), la evaluación del proceso y la evaluación "ex post".

La evaluación "ex ante": permite una aproximación a los aspectos económicos, sociales, científicos y tecnológicos de programas e instituciones, así como de centros de investigación o universidades. Requiere el análisis de resultados anteriores, juzgando las cualidades intrínsecas de un proyecto con anticipación de un grupo o de una persona, basándose en resultados anteriores.

La evaluación "ex post" (evaluation) es la relativa a los procesos de investigación ya finalizados, y permite la discusión de los resultados científicos y tecnológicos, además de los económicos y sociales relacionados con éstos. 
La evaluación de proceso (monitoring) es importante "dada su capacidad de informar durante el desarrollo e implementación de un programa. De esta manera, permite efectuar cambios o tomar medidas correctoras en función de los resultados de la evaluación continua".

Bellavista y otros asocian los tres procesos de la evaluación de la ciencia en correspondencia a los distintos tipos de objetivos (de las instituciones o de los investigadores) y con diferentes niveles de agregación: "micro", "meso" y "macro".

Vinkler (1988) establece que en el nivel "micro" los datos sobre publicaciones y citaciones investigados están referidos a un artículo, proyecto o grupo; en el nivel "meso" a un grupo de publicaciones, subdisciplina o institución y en el nivel "macro" se refieren a todas las publicaciones seleccionadas según puntos de vista dados, una disciplina en su conjunto o un grupo de países (citado por Lascurain, 2006, p. 3).

Sin embargo, para lograr una eficiente evaluación de la ciencia se requiere de indicadores biblio-ciencioinformétricos que contribuyan objetivamente en el análisis y evaluación de la información y conocimiento resultantes de la actividad científica. Estos indicadores métricos derivados de las principales disciplinas instrumentales la Bibliometría, Cienciometría e Informetría constituyen herramientas clave en la gestión de la política científica y tecnológica y en los procesos de toma de decisiones estratégicas (Arencibia, 2008, p. 2-3).

Desde hace algunos años entre las principales técnicas y métodos de evaluación de la ciencia son utilizados los llamados estudios métricos de la información, con diversos abordajes teórico-metodológicos y diferentes denominaciones en función de sus objetivos y objetos de estudio, permitiendo conocer así, la evolución de la ciencia a partir de la aplicación de técnicas de medición y trazar un perfil del mundo científico a partir de indicadores que proporcionan los estudios métricos de la información (Pires 2008, p. 122).

El desarrollo de los estudios métricos demuestra la importancia que reviste su aplicación en los diferentes contextos en que la información se pueda presentar independientemente del soporte o con el fin que es elaborada con el propósito de conocer qué elementos intervienen para la evaluación de los insumos y productos generados por la comunidad científica.

El desarrollo de los estudios métricos está enmarcado en tres disciplinas métricas principales como lo son la Bibliometría, la Informetría y la Cienciometría; aunque a lo largo de las últimas décadas varios conceptos para definirlas en función de objeto de estudio se pueden encontrar en la literatura.

Todas estas disciplinas comparten el sufijo "metría" (del griego metron, "medir"), pues todas ellas basan sus resultados en la aplicación de técnicas matemáticas y estadísticas. Y todo ello sobre la idea de base de que existe relación entre la cantidad de documentos e información con la cantidad de conocimiento (Labarta, 2011, p. 29).

A pesar de la claridad que hay al momento de establecer etimológicamente la palabra "Bibliometría", los principales autores del tema aún mantienen un gran número de desencuentros para establecer aspectos históricos, definiciones y metodologías, situación que la gran mayoría de autores reconocen; sin embargo Jiménez Contreras (2000) señala que "hay consenso en dos aspectos: en el uso que hace de métodos y modelos matemáticos, y en el objetivo que tienen de analizar conjuntos documentales, sus productores y sus consumidores" (citado por Cortés, 2007, p.47).

En este mismo orden de ideas, la Bibliometría utiliza recursos propios de los documentos permitiendo así manejar datos relativos que permitan conocer el desarrollo de las producciones intelectuales generadas en un dominio específico.

Borgman y Ferrer (2002) señalan que han surgido tres tipos de Biliometría: la descriptiva, la de relación y la evaluativa. La Bibliometría Descriptiva hace hincapié en las características de los documentos; mientras la Relacional pretende clarificar las relaciones dentro de la investigación, tales como la estructura cognitiva de los campos de investigación, la aparición de nuevos frentes de investigación, o los patrones de co-autoría nacionales o internacionales; y la Bibliometría Evaluativa, por su parte, trata de evaluar el impacto del trabajo académico, y compara las contribuciones relativas de dos o más individuos o grupos (citado por Jacobs, 2010, p. 3).

No obstante, Rubio (2004) enfatiza que la Bibliometría se divide en dos áreas: "la descriptiva y la evaluativa". La Bibliometría descriptiva trata de aspectos puramente cuantitativos, como distribución geográfica, documental, temática y su productividad. Mientras que la Bibliometría evaluativa complementa la primera con estudios de 
evaluación de la actividad científica mediante la aplicación de técnicas estadísticas y programas informáticos de mayor complejidad.

Narin (1976) resalta la importancia de la Bibliometría evaluativa porque la misma, se centra en la evaluación de la actividad científica y sobre todo en los aspectos de la calidad de sus resultados, esta concepción supera la Bibliometría descriptiva, permitiendo no sólo describir y caracterizar básicamente la producción de un país o una universidad, sino que los datos obtenidos por la evaluación se caracterizarían por su validez y fiabilidad, con una participación activa de los evaluados y con un uso efectivo de los resultados en la monitorización de la actividad científica (citados por Torres Salinas, 2007, p. 39).

Para llevar a cabo la Bibliometría y poder lograr medir los aspectos concernientes a los resultados de la actividad científica se requiere aplicar indicadores bibliométricos, que sean capaces de dar resultados objetivos acerca de los procesos de investigación y desarrollo que se llevan a cabo dentro de una comunidad científica o país.

\section{Indicadores bibliométricos para la evaluación de la ciencia como empresa social}

En el apartado anterior, se clarifica el papel que juega la Bibliometría para la evaluación de la ciencia, sin embargo, desde el punto de vista descriptivo y evaluativo debe contar con un conjunto de indicadores que permitan monitorear la actividad científica en los aspectos concernientes a: volumen, evolución, visibilidad, colaboración y estructura.

Históricamente los indicadores bibliométricos se comenzaron a emplear en la década de los treinta en los países del Primer Mundo, al respecto Licea y Arenas (2010) señalan que la actividad en algunos países se "orientó a la construcción de indicadores económicos" asimismo señala, que "a partir de la Guerra Fría la construcción de indicadores servía para comparar el potencial destructivo de las naciones" en la que años más tarde "la investigación científica, considerada una actividad social, comenzó a ser cuantificada, y comienzan a desarrollarse indicadores sociales" (p. 14).

Se entienden los indicadores como "Ios parámetros que se utilizan en el proceso evaluativo de cualquier actividad" (Sancho, 1990, p. 843). Por otro lado, la autora señala que con los indicadores se puede poner en relieve las diferentes facetas del objeto de evaluación, pero la ciencia por ser multidimensional no podrá valorarse con un indicador simple.

Sin embargo, para lograr medir y evaluar objetivamente las actividades propias de la ciencia es necesario que se apliquen indicadores bibliométricos que permitan conocer la dinámica con que se desarrolla la ciencia a partir del número de los trabajos científicos publicados, el envejecimiento de los campos científicos, la colaboración entre los científicos y las instituciones, determinar el impacto y visibilidad de las publicaciones dentro de determinada comunidad científica, entre otros.

\section{Clasificación de los indicadores}

Dentro de la literatura especializada en el área, hay una gran variedad de propuestas de autores en relación con la clasificación de los indicadores bibliométricos, tales como las de King (1987) o la de Vinkler (1998), no obstante muchos autores, a partir de estos clásicos y la dinámica de la Ciencia han ido superponiendo otros indicadores, tales como Spinak (1998) y Sancho (1990) (Torres, 2007, p.46).

Sin embargo, los trabajos realizados por Callon y Courtial (1985), Sancho (1990) y la perspectiva de Sen (1999) son algunas de las propuestas de indicadores más estudiados (Torres, 2007; Licea y Arenas, 2010): 
Tabla 1 : Indicadores bibliométricos a partir del esquema propuesto por Callon \& Courtial

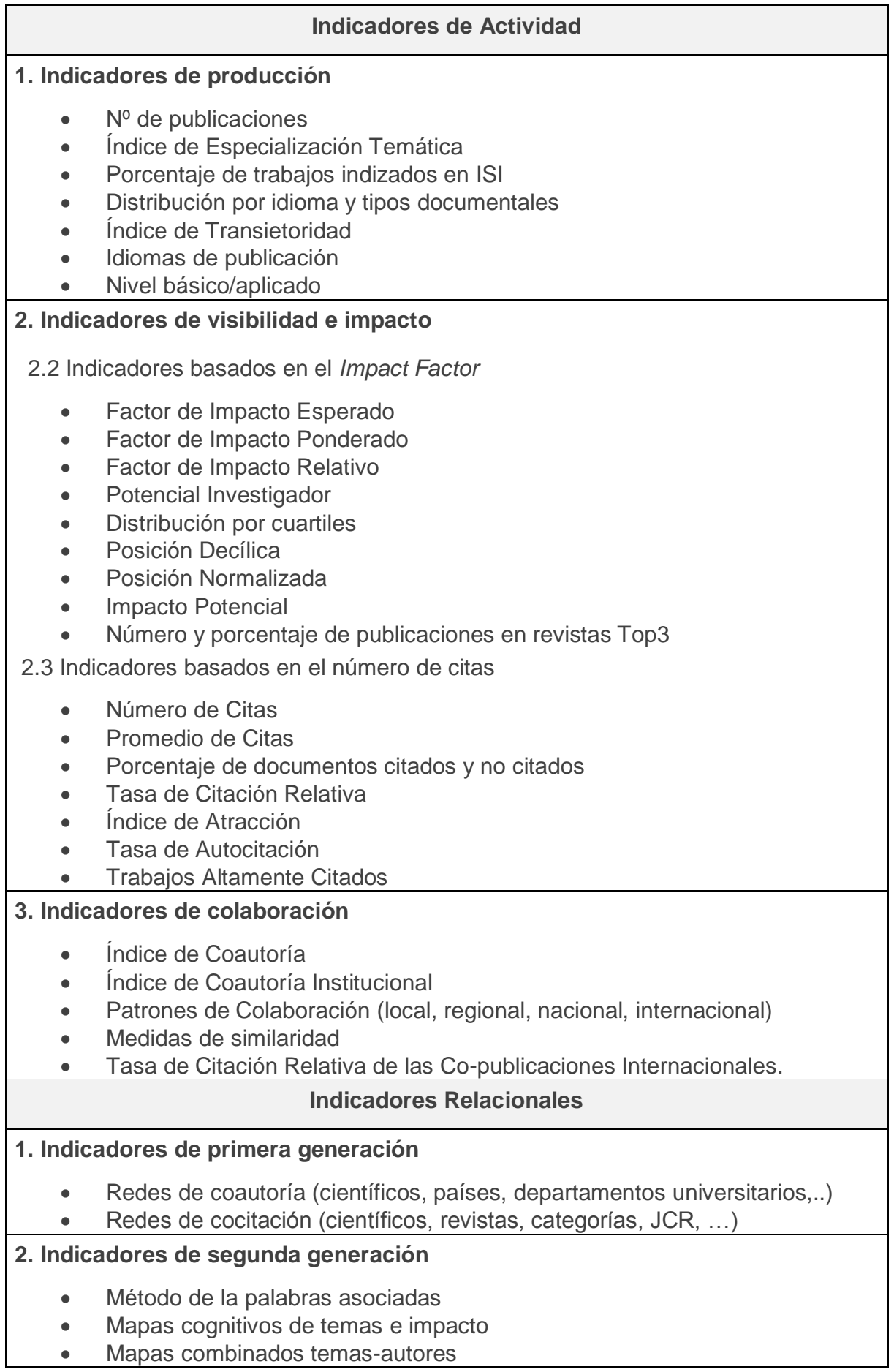

Fuente: Callon \& Courtial (1985). 
Tabla 2 : Indicadores bibliométricos a partir del esquema propuesto por Sancho

Indicadores Biblioméricos

1. Indicadores de calidad científica.

1.1 Opiniones de Expertos: indicadores basados en percepciones (peer review).

\section{Indicadores de la actividad científica.}

2.2 Número y distribución de publicaciones

2.3 Productividad de los autores: Lotka,

2.4 Colaboración en publicaciones: índice de firmas/trabajo.

\section{Conexiones entre trabajos y autores científicos}

3.1 Número y distribución de las referencias de las publicaciones científicas (reflejan los rasgos característicos del interés científico de la comunidad; Frentes de investigación y colegios invisibles; vida media, obsolescencia).

\section{Indicador del impacto del trabajo.}

4.1 Número de citas recibidas.

5. Indicadores de impacto de las fuentes.

5.1 Factor de impacto de las revistas.

5.2 Índice de inmediatez

5.3 Influencia de las revistas.

\section{Asociaciones temáticas.}

6.1 Análisis de citas comunes.

6.2 Análisis de referencias comunes.

6.3 Análisis de palabras comunes.

Fuente: Sancho (1990)

Tabla 3 : Indicadores bibliométricos a partir del esquema propuesto por Senn (continua)

Indicadores Biblioméricos

1. Indicadores derivados que son aquellos que no pueden ser calculados directamente de los documentos, pero que se preparan o calculan después de realizar algunas actividades utilizando las características e ítems implícitos en los documentos, ellos son:

1.1 Cuantificación de citas y todos los indicadores derivados del acopio de citas junto con indicadores de cita conjunta.

1.2 Indicadores calculados a partir de la frecuencia de palabras en los documentos y sus derivados, junto con indicadores basados en el análisis de palabras en común.

1.3 Categorización por tema

1.4 Todos los indicadores basados en el procedimiento de ordenamiento de revistas, países, autores, etc., de acuerdo con la productividad, cuantificación de citas, entre otros.

Fuente: Senn (1999). 
Tabla 3 : Indicadores bibliométricos a partir del esquema propuesto por Senn (conclusión)

\section{Indicadores Biblioméricos}

2. Indicadores asignados que son indicadores construidos a partir de las cualidades de los documentos o ítems bibliográficos. Algunos de ellos se dan enseguida:

2.1 Indicadores basados en los juicios de los pares.

2.2 Algunos de los indicadores acerca del uso de documentos (que pueden ser calculados a partir de la información sobre préstamo interbibliotecario, fotocopias, obtención de documentos, número de referencias, etcétera.

2.3 Indicadores sobre el análisis de la dispersión.

2.4 Clasificación de los documentos según su tema.

3. Indicadores no bibliométricos que utilizan los datos no disponibles o no pueden derivarse de la descripción del documento o de los documentos. Se trata de características relacionadas con el uso de los documentos de la biblioteca, documentos recuperados a través de una institución, número de revistas publicadas en un país, transferencia de tecnología, gasto en investigación per capita.

4. Indicadores combinados que son los que se construyen a partir de ítems bibliográficos y no bibliográficos.

Fuente: Senn (1999).

En función de las propuestas anteriores, se devela que los principales proponentes de este conjunto de indicadores encierran que los indicadores bibliométricos mayoritariamente empleados son:

1. indicadores cuantitativos de actividad científica, donde se incluyen todos los conteos de publicaciones

2. indicadores de impacto, basados en el número de citas que obtienen los trabajos, y que caracterizan la importancia de dicha producción en función del reconocimiento otorgado por otros investigadores (Bordons y Zulueta 1999; Licea y Arenas, 2010).

3. indicadores de colaboración, que parten de una de las características actuales del proceso de investigación científica, la colaboración entre autores, instituciones, países.

4. indicadores de asociaciones temáticas, que permiten establecer relaciones e identificar frentes de investigación, coocurrencia de palabras clave.

A pesar de todo lo expuesto hasta el momento, varios autores resaltan que en tiempos recientes la ciencia y la tecnología se caracterizan por la interdisciplinariedad y la transdisciplinariedad, lo cual hace que los estudios métricos de la información no resulten suficientes para describir cómo la ciencia y la tecnología afectan a las sociedades.

Directamente relacionado con el tema de la ciencia como empresa social, mucho se encuentra en la literatura en relación con la necesidad del establecimiento de nuevos indicadores que permitan medir el impacto social de la ciencia y la tecnología. Asumir que los resultados de la actividad científica deben tener una repercusión directa en la calidad de vida de los ciudadanos y en el desarrollo de los países, conduce a pensar en la necesidad de una batería de indicadores que, de conjunto con los tradicionales indicadores biblio-ciencio-informétricos reconocidos, permitan dar mayor claridad en relación con el real impacto de los resultados científicos en la sociedad. 


\section{Conclusión}

Se puede afirmar que entre las características principales de la ciencia está que es verdadera, que utiliza herramientas del pensamiento racional, que tiende a la generalidad y busca regularidades; sin embargo, la construcción de una definición de ciencia parte a partir de la realidad del contexto que vive una sociedad determinada por lo tanto la ciencia tiene un carácter dinámico y consecuentemente su significado se transforma y evoluciona a partir de los cambios que sufre la sociedad.

La ciencia como empresa social debe dar respuestas a las necesidades de la sociedad a partir de sus contribuciones. La ciencia está conformada por una comunidad científica que construye colectivamente para beneficio de un conglomerado profesional y una sociedad ávida de crecer de la mano con la ciencia desde el punto de vista tecnológico, social y educativo.

A partir de la aplicación de la Bibliometría como herramienta para la evaluación de la ciencia se pueden valorar cuantitativa y cualitativamente los elementos que constituyen el proceso de la investigación científica, para conseguirlo se requiere el empleo de indicadores bibliométricos que contribuyan objetivamente en el análisis y evaluación de la información y conocimiento resultantes de la actividad científica.

En la actualidad se debate mucho en relación con el establecimiento de un conjunto de indicadores que permitan, de conjunto con los tradicionales biblio - ciencio - informétricos medir el real impacto de la ciencia y la tecnología en la sociedad.

\section{Bibliografía}

Alcázar, E. y Lozano, A. (2009). Desarrollo histórico de los indicadores de ciencia y tecnología, avances en América Latina y México. Revista Española de Documentación Científica, [Revista en línea] 32(3). Consultado el 28 de marzo de 2013 en: http://redc.revistas.csic.es/index.php/redc/article/view/489/530

Almeida, R. (2010). Ciencia de la Información y Paradigma Social: enfoques histórico, epsitemológico y bibliométrico para un análisis del dominio. Tesis doctoral no publicada. Universidad de Granada, España.

Ander-Egg, E. (2003). Métodos y técnicas de investigación social II: la ciencia su método y la expresión del conocimiento científico. Buenos Aires: Lumen.

Arencibia, R., y Moya Anegón, F. (2008). La evaluación de la activida científica: una aproximación teórica desde la cienciometría. Acimed, [Revista en línea] 17(4). Consultado el 12/01/2013 en: http://scielo.sld.cu/scielo.php?script=sci arttext\&pid=S1024$\underline{94352008000400004}$

Becerril, A. y otros. (2012). De un modelo centrado en la revista a un modelo centrado en entidades: la publicación científica en la nueva plataforma Redalyc.org. Aula Abierta. [Revista en línea] 40(22). Consultado el 12/01/2013 en: http://dialnet.unirioja.es/servlet/articulo? codigo=3920933

Bernal, J. (1964). La ciencia de la ciencia. México: Grijalbo.

Bernal, J. (1979). Historia social de la ciencia I: la ciencia en la historia (5 ed. Vol. 1). Barcelona: Ediciones Península.

Bernal, J. (1979). Historia social de la ciencia II: la ciencia de nuestro tiempo (5 ed., Vol. 2). Barcelona: Ediciones Península.

Bordons, M., y Zulueta, M. (1999). Evaluación de la actividad científica a través de indicadores bibliométricos. Revista Española de Cardiología, [Revista en línea] 40(22). Consultado el 12/01/2013 en: http://pdf.revespcardiol.org/watermark/ctl servlet? f=10\&pident articulo=190\&pident usuario=0\&pcontactid=\&pident revista=25\&ty =19\&accion=L\&origen=cardio\&web=http://www. revespcardiol.org\&lan=es\&fichero=C521007.PDF

Bunge, M. (2004). Epistemología. [Libro en línea]. Consultado el 12 de octubre 2012 en: http://books.google.co.ve/books?id=Jti4fudh cwC\&printsec=frontcover\&dq=bunge+mario+epistemologia\&hl=es\&sa=X\&ei=7r1YUZ2 cDoGm8AS9roCABA\&redir esc $=y$

Chaviano, O. (2004). Algunas consideraciones teóricos conceptuales sobre las disciplinas métricas. Acimed, 12(4). Consultado el 12/01/2013 en: http://scielo.sld.cu/pdf/aci/v12n5/aci07504.pdf

Cortés Vargas, D. (2007). Medir la producción científica de los investigadores universitarios: la bibliometría y sus límites. Revista de la Educación Superior, 36(142). Consultado el 12/01/2013 en: http://www.redalyc.org/articulo.oa?id=60414203 
Fernández, A. (2009). El constructivismo social en la ciencia y las tecnologías: consecuencias no previstas de la ambivalencia

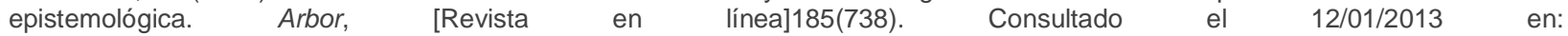
http://arbor.revistas.csic.es/index.php/arbor/article/view/324/325

Gorbea, S. (2004). Producción y comunicación científica Latinoamericana en ciencias bibliotecológica y de la información. México: Centro Universitario de Investigaciones Bibliotecológicas.

Hood, W. (2001). The literatura of bibliometrics, scientometrics and informetrics. Scientometrics. [Revista en línea], 52(2). Consultado el 07 de Julio de 2013 en: http://faculty.kfupm.edu.sa/math/kabbaj/Benchmarks/HoodWilson2001.pdf

Ísita, R. (2000). Divulgación persuasiva de la ciencia. In J. Tonda (Eds.), Antología de la divulgación científica en México [Libro en línea]. Consultado el $\quad 12 \quad$ de $\quad$ octubre $2012 \quad$ http://books.google.co.ve/books?id=VkQDfPVaTzcC\&pg=PA367\&dq=isita+tornell\&hl=es\&sa=X\&ei=n8tYUaLwHoik8ATXnlGoBw\&ve

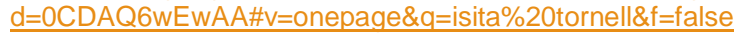

Jacobs, D. (2010). Demystification of Bibliometrics, Scientometrics, Informetrics and Webometrics. Paper presented at the 11th DIS
Annual
Conference.
Consultado
el
12
de
octubre
2012
en:

Kuhn, T. (1971). La estructuras de las revoluciones científicas. México: Fondo de Cultura Económica.

Lascurain, M. (2006). Evaluación de la actividad científica mediante indicadores bibliométricos. Bibliotecas: revista de la Escuela de Bibliotecología, Documentación e Información [Revista en línea] 24. Consultado el 29 de octubre 2012 en: http://www.revistas.una.ac.cr/index.php/bibliotecas/article/view/429

Licea, J., y Arenas, M. (2010). Estado de salud o indicadores bibliométricos en América Latina. Ciencias de la Información, [Revista en línea], 41(3). Consultado el 29 de noviembre 2012 en: http://www.redalyc.org/pdf/1814/181417704002.pdf

López Yepez, J. (1999). La evaluación de la ciencia en el contexto de las Ciencias de la Documentación. Investigaciones bibliotecológicas, [Revista en línea], 13(27). Consultado el 29 de octubre 2012 en: http://www.ojs.unam. mx/index.php/ibi/article/view/3909

Maltrás, B. (2001). Generación y comunicación del conocimiento científico. In W. Lancaster (Ed.), Procesamiento de la información científica. Madrid: Arco/Libros.

Maltrás, B. (2003). Los indicadores bibliométricos: fundamentos y aplicación al análisis de la ciencia. Gijón: Trea.

Marteleto, R. M. (2009). A pesquisa em Ciencia da Informacao no Brasil: marcos institucionais, cenarios e perspectivas Perspectiva em Ciencia da Informacao, [Revista en línea], 14 . Consultado el 29 de octubre 2012 en:http://www.scielo.br/scielo.php?pid=S141399362009000400003\&script=sci arttext

Milanés, Y., Pérez, Y., y Peralta, M. (2008). Los estudios de la evaluación de la ciencia: aproximación teórica-métrica. Acimed, [Revista en línea],18(6) Consultado el 12 de julio 2013 en: http://scielo.sld.cu/pdf/aci/v18n6/aci041208.pdf

Pacheco, J., y Milanés, Y. (2009). Evaluación de la ciencia y los estudios bibliométricos. Sirivs, [Revista en línea],1(20), Consultado el 12 de julio 2013 en: http://www.unmsm.edu.pe/veterinaria/files/evaluacion de la ciencia.pdf

Piedra, Y. (2009). La producción científica sobre comunicación: análisis desde una perspectiva de dominio en el web de la ciencia periodo 2000-2007. Tesis de Maestría no publicada, Universidad de La Habana, La Habana.

Piedra, Y., y Martínez, A. (2007). Producción científica. Ciencias de la Información, [Revista en línea], 38(3), Consultado el 22 de julio 2013 en: http://www.redalyc.org/articulo.oa?id=181414861004

Pires, D. (2008). Estudos métricos da informacao: primeiras aproximacoes. Encontros Bibli, [Revista en línea] (Número Especial), Consultado el 28 de julio 2013 en: https://periodicos.ufsc.br/index.php/eb/article/view/1518-2924.2008v13nesp1p116

Pritchard, A. (1969). Statiscal Bibliography Or Bibliometrics? Journal of Documentation, 25(4), 348-349.

Raan, A. F. J. (1996). Advanced bibliometric methods as quantitative core of peer review based evaluation and foresight exercises. Scientometrics, [Revista en línea], 36(3). Consultado el 01 de julio 2013 en: http://dx.doi.org/10.1007/BF02129602. doi:10.1007/bf02129602

Russell, J. (2004). Obtención de indicadores bibliométricos a partir de la utilización de las herramientas tradicionales de información. Paper presented at the INFO 2004. Consultado el 01 de julio 2013 en: http://www.eventos.bvsalud.org/INFO2004/docs/es/RussellJM.pdf

Sancho, R. (1990). Indicadores bibliométricos utilizados en la evaluación de la ciencia y la tecnología. Revisión bibliográfica. Revista Española de Documentación Científica, [Revista en línea],13(3), Consultado el 01 de julio 2013 en: 
http://digital.csic.es/bitstream/10261/23694/1/SAD DIG IEDCyT Sancho Revista\%20Espa\%C3\%B1ola\%20de\%20Documentacion \%20Cientifica13\%284\%29.pdf

Sanz, E., y Martín, C. (1996). Aplicación de técnicas bibliométricas a la gestión bibliotecaria. Revista General de Información y Documentación, [Revista en línea], 7(2). Consultado el 01 de julio 2013 en: revistas.ucm.es/index.php/RGID/article/download/.../10878

Sanz Menendez, L. (2004). Evaluación de la investigación y sistema de ciencia [Documento en línea], Consultado el 01 de julio 2013 en: http://www.ipp.cchs.csic.es/sites/default/files/IPP/documento trabajo/pdf/dt-0407.pdf

Torres Salinas, D. (2007). Diseño de un sistema de información y evaluación científica. Análisis cienciométrico de la actividad investigadora de la Universidad de Navarra en el área de ciencias de la salud 1999-2005. [Tesis Doctoral en línea], Universidad de Granada, Granada. Consultado el 01 de julio 2013 en: http://hera.ugr.es/tesisugr/16615645.pdf

\section{Datos de los autores}

\section{Guillermo Andrés Pérez Reyes}

Master en Bibliotecología y Ciencias de la Información. Egresado de la Universidad de la Habana, Facultad de Comunicación, La Habana, Cuba. Licenciado en Bibliotecología y Ciencias de la Información por la misma universidad. Jefe de la Biblioteca de la Universidad Politécnica Territorial Andrés Eloy Blanco Barquisimeto, Venezuela. Profesor Instructor del Programa de Formación en Ciencias de la Información de la Universidad Politécnica Territorial Andrés Eloy Blanco, Barquisimeto, Venezuela. Integrante del Comité Interinstitucional para el Fortalecimiento de la Gestión del Programa Nacional de Formación en Ciencias de la Información, Ministerio del Poder Popular para la Educación Universitaria, Caracas, Venezuela.

pguillermoandres@gmail.com

\section{Ailín Martínez Rodríguez}

Master en Bibliotecología y Ciencias de la Información. Egresada de la Universidad de la Habana, Facultad de Comunicación, La Habana, Cuba. Licenciada en Bibliotecología y Ciencias de la Información por la misma universidad. Profesora Principal Auxiliar de la carrera Bibliotecología y Ciencia de la Información de la Facultad de Comunicación en la Universidad de la Habana. Ha participado en eventos nacionales e internacionales como ponente y como invitada. Ha publicado artículos en revistas indexadas en bases de datos internacionales. Se ha desempeñado como Jefa del departamento docente de Bibliotecología y Ciencia de la Información de la Universidad de la Habana.

ailin.martinez@infomed.sld.cu

Recibido - Received: 2013-12-04

Aceptado - Accepted: 2014-06-30

\section{$(c))_{E Y}$}

This work is licensed under a Creative Commons Attribution 4.0

United States License.

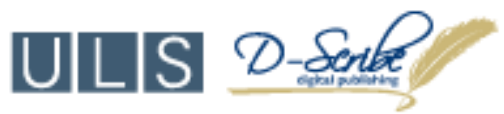

This journal is published by the University Library System of the University of Pittsburgh as part of its D-Scribe Digital Publishing Program and is cosponsored by the University of Pittsburgh Press. 